OPEN ACCESS

Edited by:

Vito Di Maio,

Istituto di Scienze Applicate e Sistemi Intelligenti "Eduardo Caianiello"

(ISASI), Italy

Reviewed by:

Henrique Prado von Gersdorff, Oregon Health \& Science University,

United States

Eric Hanse

University of Gothenburg, Sweden

*Correspondence:

David Holcman holcman@biologie.ens.fr

Received: 01 February 2018 Accepted: 29 June 2018 Published: 23 July 2018

Citation:

Guerrier C and Holcman D (2018) The First $100 \mathrm{~nm}$ Inside the Pre-synaptic Terminal Where Calcium Diffusion

Triggers Vesicular Release.

Front. Synaptic Neurosci. 10:23. doi: 10.3389/fnsyn.2018.00023

\section{The First $100 \mathrm{~nm}$ Inside the Pre-synaptic Terminal Where Calcium Diffusion Triggers Vesicular Release}

\author{
Claire Guerrier ${ }^{1}$ and David Holcman ${ }^{2,3 *}$ \\ ${ }^{1}$ Department of Mathematics and Brain Research Center, University of British Columbia, Vancouver, BC, Canada, ${ }^{2}$ Group of \\ Applied Mathematics and Computational Biology, IBENS, École Normale Supérieure, Paris, France, ${ }^{3}$ Churchill College, \\ Cambridge University, Cambridge, United Kingdom
}

Calcium diffusion in the thin $100 \mathrm{~nm}$ layer located between the plasma membrane and docked vesicles in the pre-synaptic terminal of neuronal cells mediates vesicular fusion and synaptic transmission. Accounting for the narrow-cusp geometry located underneath the vesicle is a key ingredient that defines the probability and the time scale of calcium diffusion to bind calcium sensors for the initiation of vesicular release. We review here the time scale, the calcium binding dynamics and the consequences for asynchronous versus synchronous release. To conclude, three-dimensional modeling approaches and the associated coarse-grained simulations can now account efficiently for the precise co-organization of vesicles and Voltage-Gated-Calcium-Channel (VGCC). This co-organization is a key determinant of short-term plasticity and it shapes asynchronous release. Moreover, changing the location of VGCC from few nanometers underneath the vesicle modifies significantly the release probability. Finally, by modifying the calcium buffer concentration, a single synapse can switch from facilitation to depression.

\begin{abstract}
Keywords: synaptic transmission, stochastic modeling, vesicular release, asynchronous release, facilitation, short-term plasticity (STP), residual calcium, simulations
\end{abstract}

\section{INTRODUCTION}

The first $100 \mathrm{~nm}$ domain, between the plasma membrane and the vesicles in the pre-synaptic terminal remains difficult to study, yet it seems that a displacement as small as a 10 of nanometers in the molecular organization can affect vesicular release. There are many examples, where a 10 $\mathrm{nm}$ precision has to be achieved in order to guarantee normal physiology function. This is the case for the apposition of pre- and post-synaptic terminal of neuronal synapses: this apposition is obtained by a set of redundant adhesion molecules, such as laminins that self-organize to maintain the synapse structure and stability in the central nervous system. A lack of the laminin $\beta_{2}$ subunit leads to a disruption of the hippocampal synapse structure, to a misalignment of the pre- and post-synaptic partners and to an increased post-synaptic density (PSD) size (Egles et al., 2007). In addition, mutations in PSD proteins are associated with neurological and psychiatric diseases (Sheng and Kim, 2011). Another example is autism spectrum disorders which have been associated with the mutations in genes encoding Shank2 and Shank3, PSD-93, and a mis-regulation of adhesion molecules neuroligin 3, neuroligin 4, and neurexin 1 (Durand et al., 2011; Sheng and Kim, 2011), affecting a precise geometrical apposition. 
In the pre-synaptic terminal, some vesicles are concentrated in a region called the Active Zone (AZ), which is well-aligned with the PSD of the post-synaptic terminal. This apposition creates geometrical columns: a single column alignment was originally hypothesized and numerical simulations showed that it maximizes the synaptic current (Xie et al., 1997) while minimizing its fluctuations (Taflia and Holcman, 2011). Multiple nanocolumns were predicted in Freche et al. (2011) to sustain synaptic response and a more reliable transmission compared to several synapses containing a single column. Finally, these columns have recently been confirmed experimentally and observed at super-resolution (Tang et al., 2016; Biederer et al., 2017). This nanocolumn example shows that synaptic transmission uses a tens of nanometer precision for its organization and for example a misalignment of synaptic terminals is at the basis of several pathological disorders (Südhof, 2008).

Another example is the PSD, that cannot permanently retain glutamatergic receptors that are moving by random motion (Nicoll, 2017). After a long enough time, these receptors spread out, modifying the synaptic current (Freche et al., 2011; Fresche et al., 2013). In the absence of direct experimental approaches, studying the functional consequences of the nanometer precision in the domain between the membrane and the vesicles at the presynaptic terminal has recently benefited from three-dimensional modeling and numerical simulations. We focus this review on this paradigm shift of analyzing the diffusion of calcium ions and in particular about the nano-metric relation between the organization of calcium channels and vesicles and how it shapes the release probability, synaptic transmission, asynchronous release, and short-term plasticity.

\section{DYNAMICS AND CONSTANT RE-ORGANIZATION IN THE PRE-SYNAPTIC TERMINAL}

Despite the fast advances of super-resolution microscopy, that allowed to reconstruct structural in vivo cell properties, or to follow calcium and voltage using genetically encoded indicators (Holcman and Yuste, 2015; Popovic et al., 2015; Beaulieu-Laroche and Harnett, 2018; Cartailler et al., 2018), it remains difficult to study the detailed molecular dynamics in nanometer domains at a time scale $<100 \mathrm{~ms}$. Indeed, to understand how molecules interact in nanometer domains, the notion of concentration has to be abandoned because it does not make much sense due to the large fluctuations in the small number of molecules. However, molecular interactions can still be transformed into a cellular activation at the micrometer level, but the exact biophysical mechanisms remain in most cases unclear or controversial. Modeling and numerical simulations based on biophysical principles have emerged as orthogonal tools compared to experiments to describe molecular dynamics at this spatio-temporal scales (Holcman and Schuss, 2015).

At this intermediate level between the molecular and the cellular scale, physical modeling of diffusion is based on
Brownian motion, which requires to specify an inherent time scale of simulations. Indeed the motion of molecules follows a random walk approximation expressed by the Euler's scheme for a trajectory $\boldsymbol{X}(t)$ at time $t$ :

$$
\boldsymbol{X}(t+\Delta t)=\boldsymbol{X}(t)+\sqrt{2 D(\boldsymbol{X}) \Delta t} \eta
$$

where $\boldsymbol{\eta}$ is a Gaussian variable, $D(\boldsymbol{X})$ is the spatial dependent diffusion coefficient and $\Delta t$ is the time scale to be chosen. It is usually a difficult choice. It should not be too small to avoid wasting simulation times and should not be too large compared to the small spatial scales involved in the microdomain, such as molecular binding sites.

In particular, taking into account in numerical simulations the region between vesicles and the plasma membrane has been particularly difficult to model because of its cusp-like geometry. It requires a specific mathematical treatment to estimate the mean time for a calcium ion after entering through a Voltage-Gated-Calcium-Channel (VGCC) to find a key calcium binding sensor, involved in triggering vesicular release (Guerrier and Holcman, 2014) (Figure 1). Such sites are $\mathrm{Ca}^{2+}$-binding proteins, located on synaptotagmins, that are involved in triggering directly or not vesicular fusion (Lee and Littleton, 2015). They are located precisely in this nanometric region below vesicles. Interestingly, spontaneous excitatory and inhibitory transmission are differently regulated by $\mathrm{Ca} 2+$ sensors (synapotagmin-1 and Doc $2 \alpha / \beta$ a high-affinity $\mathrm{Ca} 2+$ sensors) (Courtney et al., 2018).

Calcium diffusion in the pre-synaptic terminal has traditionally been modeled as two- or three- dimensional diffusion (Modchang et al., 2010; Nadkarni et al., 2010; Stanley, 2016), but ignoring the three-dimensional complications of the vesicle shape. However, for auditory hair cells, Monte-Carlo simulations revealed (Graydon et al., 2011) that the spherical shape of the ribbon where vesicles are tethered, can generate a local $\mathrm{Ca}^{2+}$ microdomain that enhances vesicular fusion by trapping calcium ions (Graydon et al., 2011, Figure 3B). This spherical ribbon that aggregates vesicles is likely to create an intermediate microdomain for calcium dynamics between the pre-synaptic bulk and the boundary layer near the membrane, which should be further investigated. For other types of synapses, while estimating the time scale of calcium binding, and computing the vesicular release probability, one cannot ignore the specific three-dimensional organization of the first $100 \mathrm{~nm}$, the region underneath vesicles and the position of calcium sensors (Rothman et al., 2017). The release probability not only depends on the binding of calcium ions to sensor proteins, located underneath the vesicle (Guerrier and Holcman, 2015), but also on the AZ organization: a sparse vesicular distribution vs. vesicular crowding, and channels clustered vs. uniformly distributed (Figure 1B) (Delvendahl et al., 2015; Nakamura et al., 2015). The major components in these dependencies being the distance between VGCC and vesicles coupled to the particular cusp-like geometry (Guerrier and Holcman, 2015). 
A

C

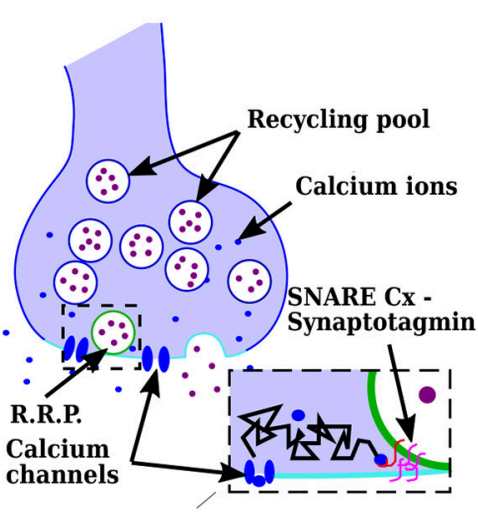

B

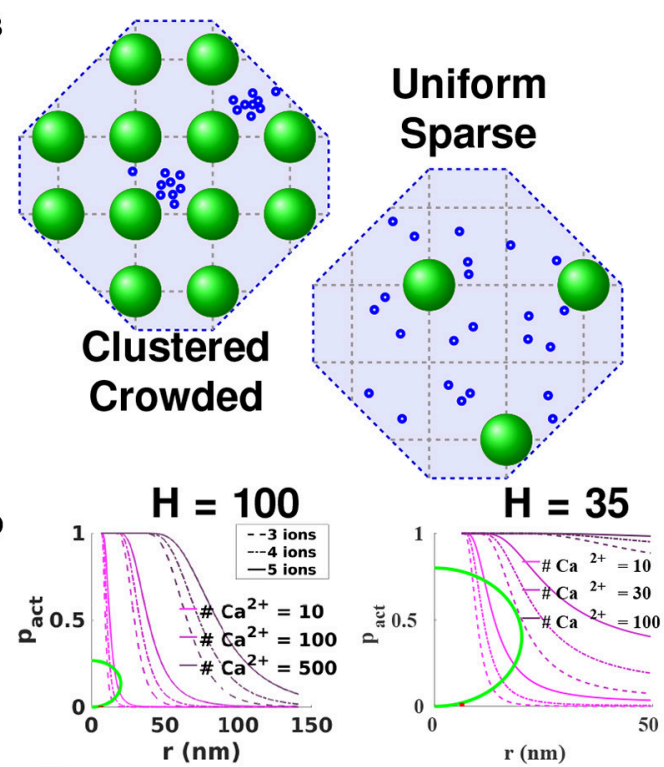

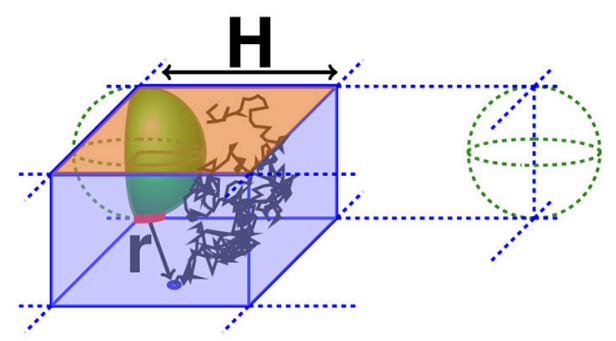
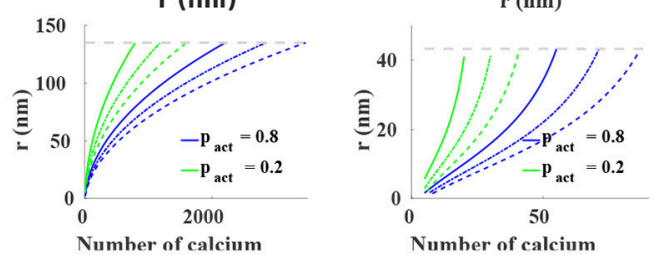

FIGURE 1 | Estimating the release probability. (A) Functional organization of the presynaptic terminal. An incoming action potential leads to the opening of voltage gated calcium channels (blue) located at the AZ (light blue). The consecutive entry of calcium ions (orange) triggers the fusion of docked vesicles (green) with the synaptic membrane, and the liberation of neurotransmitters (purple) in the synaptic cleft. The binding of neurotransmitters to specific receptors located in the post-synaptic terminal triggers the conversion of the chemical signal into an electrical signal in the post-synaptic neuron. (B) Model of the AZ organization. Vesicles (green) are regularly (left) or sparsely (right) distributed on a square lattice. Calcium channels (blue) can be clustered (left) or uniformly distributed (right) in the AZ.

(C) Elementary 3-dimensional domain to compute the splitting probability for an ion starting in the bottom of the domain (blue circle representing a channel), to reach the target (red) before leaving the domain through the orange boundary. The other boundaries are reflecting. The vesicles are distributed on a square lattice of side $2 \mathrm{H}$. (D) (Top): Probability to find three, four, or five calcium ions (full, dashed and dotted lines respectively) underneath a vesicle, in the case of sparse vesicular distribution: $H=100 \mathrm{~nm}$ (left) and in the case of crowding of vesicles at the AZ: $H=35 \mathrm{~nm}$ (right). The relation depends on the initial number of calcium ions. The diameter of the pre-synaptic vesicles is fixed at $R=40 \mathrm{~nm}$ (green), the diffusion coefficient for free calcium ions being $D_{\mathrm{Ca}}=200 \mu \mathrm{m}^{2} \mathrm{~s}^{-1}$. (Bottom): Maximal channels distance $r$ to activate the vesicle with a probability $p_{\text {act }} \geq 0.8$ (blue) and 0.2 (green), when there are $N$ initial ions, for $H=100 \mathrm{~nm}$ (left) and $H=35 \mathrm{~nm}$ (right). We fix the threshold to three, four, or five calcium ions. The gray dashed line represents the maximal distance to the vesicle in the elementary domain: $\sqrt{2} H$.

\section{CALCIUM BINDING SENSORS AND VESICULAR RELEASE KINETICS}

The synaptotagmin family of molecules are $\mathrm{Ca}^{2+}$ sensors for vesicle fusion: following $\mathrm{Ca}^{2+}$ binding, activation of the SNARE-complex mediates membrane fusion. Although there are two specific sensors on synaptotagmin, the number of ions necessary for fusion was estimated around 4 or 5 (Kochubey et al., 2011; Schneggenburger et al., 2012). Indeed, for example synaptotagmin 1 has two $\mathrm{Ca} 2+$ binding $\mathrm{C} 2$ domains (C2A and $\mathrm{C} 2 \mathrm{~B})$ and three $\mathrm{Ca} 2+$ bind in $\mathrm{C} 2 \mathrm{~A}$ and two $\mathrm{Ca} 2+$ bind in $\mathrm{C} 2 \mathrm{~B}$ (Gruget et al., 2018).

\subsection{Modeling Calcium Binding and Limitation of Using a Forward Rate Constant}

Modeling the causality between calcium dynamics and vesicular fusion relies on patching steps, resulting in simulations that do not necessarily account for the three-dimensional organization of the AZ. In Keller et al. (2015), by using the software Mcell, the forward rate constant of calcium ions is the reciprocal of the flux to the sensor targets is assumed rather than derived from physical considerations. This rate has to be pre-calculated especially when calcium sensors are located underneath vesicles (see next section). In addition, due to the vesicle crowding, the calcium flow cannot be represented by a Gaussian function, which is the classical probability density function of independent particles initially concentrated at one spot in a free space. This approximation further neglects the boundary effect and the depth of a synapse. In that context, providing numbers such as a distance of $30 \mathrm{~nm}$ for a possible exclusion area between vesicles and VGCCs, based on a two-dimensional approximation that ignores the effect of the vesicle size with a radius of $R=20$ $\mathrm{nm}$ is not necessarily accurate. But the question is by how much? Clearly this geometrical limitation calls for a three-dimensional approach accounting for vesicular structure and organization. 
A different modeling approach is described in Nakamura et al. (2015), based on diffusion (Nielsen et al., 2004; DiGregorio et al., 2007) of a two-dimensional coarse-grained lattice, using a two-dimensional Gaussian approximation for the initial calcium entrance. The vesicular release in these approaches is computed by using a Markov model, which is based on the concentration of calcium in a two-dimensional domain around the location of the channel. However, this concentration does fluctuate a lot, depending on the size of the sampling volume. This modeling approach neglects the small number of ions that penetrate underneath the vesicle, which is replaced by assuming a value for the forward rate. Indeed, to trigger vesicular release from the binding of calcium ions to sensors, a value should be given to the forward rate $k_{o n}$. Since the early work of von Smoluchowski in 1916 (Holcman and Schuss, 2017), this rate has been computed as the flux from a fixed concentration to a narrow window located on an infinite plan. But these assumptions are not satisfied for the small steady-state calcium concentration, because the rate should be computed for the first calcium arriving to the binding site, sampled from the transient entrance through the VGCC (Basnayake et al., 2017).

In summary, using a forward rate constant presuppose a geometrical organization. An alternative is to replace such a rate by Brownian simulations, but this approach is in general heavy computationally. Recent hybrid simulations have been developed, where classical diffusion is used far away from a sensor, while near the boundary of a channel a Brownian representation is used (see Dobramysl and Holcman, 2018 for a description of such framework).

\subsection{How to Chose an Effective Diffusion Coefficient}

What should be the buffer distribution and concentration in the pre-synaptic terminal? Various buffers and concentrations were previously considered: for the calyx of Held, a concentration of $400 \mu M$ immobile endogenous buffers plus a Parvalbumin-like $\mathrm{Ca}^{2+}$ diffusing buffer with concentration of $50 \mu M$ were used in Keller et al. (2015), while ATP, a mobile $\mathrm{Ca}^{2+}$ buffer, was present at a total concentration of $2 \mathrm{mM}$ in all simulations (with a diffusion coefficient of $220 \mu \mathrm{m}^{2} / \mathrm{s}$ ). In Delvendahl et al. (2015) the concentration of fixed buffers is $480 \mu M$, and of mobile buffers is $100 \mu M$ with $D=20 \mu \mathrm{m}^{2} / \mathrm{s}$. The buffer capacity $\kappa_{S}$ (ratio of bound vs. free) is often chosen equal to 40 . However, one of the main free parameter remaining is the value of the forward rate, which is valid for a single compartment model like the presynaptic terminal, which disregard its heterogeneity, but not for buffers located underneath vesicles.

\subsection{Calcium-Buffer Interactions in the Pre-synaptic Bulk}

When the pre-synaptic terminal is modeled as a bulk only, it does not matter that buffers are moving or not, because in one compartment the differential equations disregard the geometry. So, what matters is the number of free buffers available. If buffers are modeled with stochastic simulations, then space matters, especially during multiple entry of calcium channels, due to fluctuations of calcium buffers in the region very close to the calcium sensor sites underneath the vesicle. But to be efficient, this geometry should be implemented, which is often difficult.

In summary, buffers could be homogeneously distributed in the bulk, but between vesicles, the concentration is much less homogenous. More drastically, the number of buffers between the membrane and the first layer of vesicles can be of the order of a few: indeed, for vesicles positioned on a square lattice with radius $60 \mathrm{~nm}$ with a height of $40 \mathrm{~nm}$, the volume of the parallelepiped lattice $P_{\text {para }}$ is $V=0.06 \times 0.06 \times 0.04=24 \times$

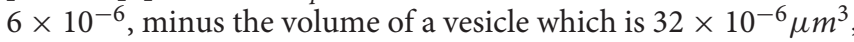
that is $\mathrm{Vol}_{\text {total }}=1.12 \times 10^{-4} \mathrm{\mu m}^{3}$. Inside such a region, for a buffer concentration of $40 \mu M$, this represents around 26 buffers. For $400 \mu M$ (at the calyx of Held), this represents around 260 buffers. These numbers should be compared to the number of free calcium entry (from 80 to 500 ). It is conceivable that the different vesicular proteins located near the vesicular calcium sensor (others than the synaptotagmins) play a more important role for buffering calcium than the diffusing calcium buffers located in the bulk which can occasionally enter into the region $P_{\text {para }}$, because they are precisely located at the right place and thus could create an efficient local calcium reservoir.

Finally, calcium mitochondria uptake can affect synaptic release through the MCU channels (Kwon et al., 2016). Mitochondrias participate in the calcium regulation that controls synaptic release and a MCU disruption could increase asynchronous release, decreasing the efficacy of synchronous neurotransmitter release and could also alter short-term presynaptic plasticity. This suggests that the distribution of mitochondria within the $\mathrm{AZ}$ could be as determinant as calcium buffers, a question that should be further investigated.

Another reason to reconsider the role of calcium buffer in the first $100 \mathrm{~nm}$ layer is the presence of an electric field that could push ions inside the bulk, as revealed recently for the synaptic terminal (Cartailler and Holcman, 2018; Cartailler et al., 2018). Too many buffers should disrupt calcium signaling and direct vesicular release. A low buffer capacity will increase asynchronous release. Thus, the concentration of buffers that favor a synchronous release should have an optimal value: not too low and not too large.

In Guerrier and Holcman $(2015,2016)$, the number of calcium entering through the VGCC vary from 80 to 500 , which is also the case in Delvendahl et al. (2015), where they open around $12 \mathrm{Ca}^{2+}$ channels with a single channel current of $0.15 \mathrm{pA}$ and a duration of $105 \mu$ s, leading to $\approx 500-600$ calcium ions. Some of the 260 buffers with at least two binding sites, could bind calcium ions on their direct way to the calcium sensor underneath the vesicle. With ten times less buffers, much more free calcium would be available and then what would matter is the distance of the channel to the vesicle.

\subsection{Phenomenological Laws Between Probability and the Overall Calcium Concentration}

The relation between the molecular organization of VGCC, their numbers, calcium buffer dynamics, the release probability $P_{\text {release }}$ and the calcium flux of entering concentration of calcium $[\mathrm{Ca}]_{f l u x}$ 
mediated by an action potential remains an interesting problem. Over the years the following empirical relation has been proposed (Kochubey et al., 2011):

$$
P_{\text {release }}=k\left([\mathrm{Ca}]_{f l u x}\right)^{a},
$$

where $k$ is a constant. The relation between the exponent $a$ and the number of active sensor binding sites is not direct, due to the effect of buffers, the clustering of VGCCs, but also the local vesicular geometry. Indeed, the contribution of geometry appears while computing the probability for an ion to go underneath the vesicle vs. going directly to the bulk (Guerrier and Holcman, 2014; Keller et al., 2015).

\subsection{Partial Conclusion: Modeling Vesicular Release}

To conclude, there is not yet a derived formula from physical principle to connect the flux or transient calcium concentration and the release probability, however, stochastic approaches are used to estimate the arrival of ions to the calcium sensors (Delvendahl et al., 2015; Guerrier and Holcman, 2015, 2016; Nakamura et al., 2015). In addition, the three dimensional vesicular organization should be accounted either directly, by implementing vesicles as obstacles or by computing the Brownian flux to small targets located underneath.

Following calcium diffusion, once calcium ions are bound to buffers, they can possibly unbind, but often the exact value of the backward rate constant is unknown. In recent mathematical models (Guerrier and Holcman, 2015, 2016; Keller et al., 2015), vesicles are released when all 5 binding sites at a single sensor are occupied. If less than 5 calcium ions are bound, the vesicle is waiting for the final ions to arrive. There can be several copies of molecular sensors, but a single one might be sufficient to trigger release. It might also be conceivable that multiple binding sensors cooperate in the release process, and this possibility could explain the large modulation of the vesicular release probability (Kochubey et al., 2011; Schneggenburger et al., 2012).

We already emphasized that the calcium ions bound to calcium sensors located underneath the vesicles can contribute critically to the residual calcium ions pool, especially when the backward rate is very small. To conclude, there are two types of calcium ions contributing to shaping the vesicular release probability. The ions already bound to the specific calcium sensors located underneath the vesicles, and the ions freely moving in the pre-synaptic terminal, that can reach the calcium sensors or induce calcium release from organelles, hence filling a binding site, and ultimately triggering vesicular fusion. This mechanism represents a possible scenario for the calcium contribution to the asynchronous vesicular release (see below).

\section{DISTRIBUTION OF CALCIUM IONS ENTERING THROUGH A VGCC}

The Hodgkin-Huxley model (Hodgkin and Huxley, 1952) can be used Guerrier and Holcman (2016) to generate a calcium influx current inside the pre-synaptic terminal. Following the opening of VGCC, this current corresponds to an entry per channel during a mean time of $\approx 1 \mathrm{~ms}$ for approximately 80 calcium ions, compatible with a previous estimation of 200 reviewed in Stanley (2016) or with the 45 ions per channels described in Keller et al. (2015): with a total of 12 channels, this would represent 540 ions entering during $\approx 0.1 \mathrm{~ms}$. After entry, the calcium flux can be split into a ionic component that reaches the small region below the vesicle, and another one reaching the pre-synaptic bulk. The probability for a calcium ion to reach the binding region, defined as a small ribbon joining the vesicle and the plasma membrane, before the bulk, has been computed in models where vesicles are organized in a square lattice (Figure 1B) with length $2 H$, where $H$ is of the order of the diameter of a vesicle, from 40 to $100 \mathrm{~nm}$ (Figure 1C). For a dense set of vesicles distributed on a square lattice, the splitting probability for a calcium ion (modeled as Brownian) to reach the ribbon before the bulk is (Guerrier and Holcman, 2015):

$$
p_{s}(x)=1-\frac{1-A \frac{r_{v}^{2} \varepsilon}{H^{3}}}{1-\frac{2 r_{v} \varepsilon}{H^{2}}}\left(1-\frac{2 r_{v} \varepsilon}{r(x)^{2}}\right),
$$

where $r_{v}$ is the size of a vesicle, $H$ is half the distance between two vesicles, $A=9.8, r(x)$ is the distance between the point source and the closest vesicle, and $\varepsilon$ is the height of the small cylindrical ribbon (Figure 1C), where calcium sensors are located. This probability accounts for the particular geometry of the target and depends on the relative distance between the targets and the source points (Guerrier and Holcman, 2015).

\subsection{Calcium Time Scales to the Ribbon Region}

How long does it take for a calcium ion in the synaptic bulk or at the mouth of a VGCC (located far away from a vesicle) to enter into the cylindrical ribbon (red region in Figures 1A-C) underneath a vesicle? This mean time computed analytically in Guerrier and Holcman (2015) and Holcman and Schuss (2015) is given by:

$$
\bar{\tau}=\frac{|\Omega|}{4 \pi D \varepsilon} .
$$

For a volume of a pre-synaptic microdomain $|\Omega|=1 \mu \mathrm{m}^{3}$, a diffusion coefficient $D=20 \mu \mathrm{m}^{2} / \mathrm{s}$, and a size of the ribbon in the range $\varepsilon=0.001-0.01 \mu \mathrm{m}$, the mean time is $\bar{\tau}=$ 3.6s. We note that the diffusion coefficient of calcium ions in a free environment is usually $D \approx 200 \mu \mathrm{m}^{2} / \mathrm{s}$, but the motion of ions in pre-synaptic terminals or in dendrites is restricted by obstacles such as microtubules, actin and organelles. In Biess et al. (2011), the effects of crowding on the diffusion coefficient has been estimated using modeling, simulations and a cytoplasmic fluid in a patch pipette, leading to a modified effective diffusion coefficient $D \approx 20 \mu \mathrm{m}^{2} / \mathrm{s}$.

However, the size of a calcium ion of the order of $1 \mathrm{~nm}$ should not affect the classical law of diffusion. So the size of a calcium ion is often neglected in most modeling and stochastic simulations. Certainly, the most interesting part of vesicular crowding microenvironments is the local molecular organization underneath 
the vesicle, formed by all vesicular molecules, such as SNARE, syntaxin, or synaptotagmin, that could result in a 10-20 nm environment filled with polymer filaments. This intermediate spatial scale could have several effects such as (1) sequestrating calcium ions and/or creating channels to any sensor sites, (2) preventing calcium channels to get too close to the calcium sensors, (3) positioning synaptotagmin close to VGCC.

To conclude, as crowding is the main obstacle to diffusion, when the diffusion time scale involves long distances the effective diffusion coefficient should be used, while for short-distances containing little obstacles, computation should be performed with the cytoplasmic diffusion coefficient. No evidences have shown that changes of the cytoplasmic volumes, occurring at a time scale of milliseconds can modify the nature of calcium diffusion.

\subsection{Direct and Indirect Vesicular Release Activation}

Using the values mentioned in the previous subsection, the mean time for calcium to transit from the bulk underneath a vesicle is $\bar{\tau}=\frac{1}{k_{S}}=\frac{|\Omega|}{4 \pi D \varepsilon}=3.6 \mathrm{~s}$ (for $\left.\varepsilon=1 \mathrm{~nm}\right)$ to $360 \mathrm{~ms}$ (for $10 \mathrm{~nm}$ ). This mean time is much longer than the initial calcium transient from the channels $(<5 \mu s)$, as we shall see now. The reciprocal of this time is the Poissonian rate $k_{S}$ representing the rate of arrival of a free calcium ion to a binding sensor. However, this time is very different from the time for an ion entering through a VGCC close to the vesicle, to reach the region underneath the vesicle directly, i.e. while staying in a boundary layer around it. Indeed due to the confinement by the vesicle and in the absence of large obstacles at a distance of $20 \mathrm{~nm}$, the time for an ion to hit a target sensor is $\tau_{\text {direct }} \approx \frac{l^{2}}{4 D}$. Using the free diffusion coefficient for calcium $D \approx 200 \mu \mathrm{m}^{2} / \mathrm{s}$, this leads to a mean time of $\tau_{\text {direct }} \approx$ $\frac{0.02^{2}}{800}=0.5 \mu \mathrm{s}$. Thus when the channel is located very close to the vesicle, the direct binding of a single calcium is not time limiting. However, when the distance increases, the mean time for 5 calcium ions to arrive from a channel can be much longer (Guerrier and Holcman, 2015) of the order of 5-500 $\mu$ s, due to large fluctuations in the arrival time, the accumulation time for 5 ions (Dao Duc and Holcman, 2010) and the fast transient in the calcium available at the entrance of the channel. This scenario provides a physical mechanism for the fastest transmission events reported in Sabatini and Regehr (1996) and Von Gersdorff and Borst (2002).

\section{COMPUTING THE RELEASE PROBABILITY WHEN VGCC ARE LOCATED UNDERNEATH A VESICLE}

The probability that a finite number $T$ of calcium ions $(T=3,4$, and 5 ions) are bound at specific binding sites located between a vesicle and the synaptic membrane (section 3.1), when $N$ calcium ions have entered through a cluster or a single VGCC located at a distance $r$ from the center of the closest vesicle is defined by:

$p_{\text {act }}(r, N)=\mathcal{P}(T$ ions have reached the synaptotagmin $\mid N$ ions, distance $r$ ).
When the calcium unbinding events are too slow to be taken into account, the probability of vesicular release $p_{a c t}(r, N)$ is thus the one to find at least $T$ ions inside the cylindrical ribbon. The probability to find exactly $k$ ions out of $N$ follows the Binomial distribution $\mathcal{B}\left(N, p_{s}(r)\right)$, and the steady-state probability is:

$$
\begin{aligned}
p_{a c t}(r, N) & =\sum_{k \geq T}\left(\begin{array}{l}
N \\
k
\end{array}\right) p_{s}(r)^{k}\left(1-p_{s}(r)\right)^{N-k} \\
& =1-\sum_{k=0}^{T-1}\left(\begin{array}{l}
N \\
k
\end{array}\right) p_{s}(r)^{k}\left(1-p_{s}(r)\right)^{N-k},
\end{aligned}
$$

where $p_{s}(r)$ was computed in Equation (3). The maximal VGCC distance $r_{\text {max,pact }}(N)$ to activate a vesicle with a probability $p_{\text {act }} \geq 0.8$ is shown in Figure 1D. The probability pact critically depends on the channels-vesicles distance, which can vary from a few to hundreds of nanometers. This fast decrease of the probability with the distance explains the large variability in the release probability as VGCC position can vary over time (Schneider et al., 2015).

The organization of vesicles in the $\mathrm{AZ}$ also influences the release probability: when vesicles are sparsely distributed (Figures 1B-D, $H=100 \mathrm{~nm}$ ) and 100 ions entered through VGCC, then a $80 \%$ release probability $p_{a c t}=0.8$ is reached when the distance between the vesicles and the channels is smaller than $24 \mathrm{~nm}$, compared to the $20 \mathrm{~nm}$ radius of the vesicle. This result shows that the co-localization of VGCC with a vesicle is a key feature determining a high synchronous release probability. However, for a high vesicular crowding (Figures 1B-D, described by choosing the distance $H=35 \mathrm{~nm}$ ) and when 100 ions are released instantaneously at a VGC, then the probability $p_{a c t}$ is higher than 0.9 , regardless of the initial position of the channel, suggesting that vesicles are certainly released and leading to a synchronous release.

To conclude, a high crowding of vesicles should be associated with a high-release probability sustaining a synchronous release, while a sparse vesicle density might be associated with asynchronous release. Channels can be organized in clusters or uniformly distributed and this is also a major determinant governing release probability (Figures 1B-D). Indeed, the effect of channels clustering can be modeled by simply increasing the number of entering calcium ions. When vesicles are sparsely distributed, the $24 \mathrm{~nm}$ distance required to obtain a release probability $p_{a c t}=0.8$ when 100 ions are entering through one channel, is increased to $61 \mathrm{~nm}$ for 500 ions. This effect results from the local geometry of the ribbon underneath the vesicle. When the number of ions is low, this maximal distance to guarantee $p_{\text {act }}=0.8$ does not vary much when the activation threshold $T$ increases from 3 to 5 ; however, for 500 ions, this distance changes significantly over 15 nm.

The maximal distance $r_{\max , p_{a c t}}(N)$ between channels and vesicles to obtain a given release probability $p_{a c t}$ depends on the number $N$ of entering ions. For a fixed probability $p_{a c t}$, we plotted $r_{\max , \text { pact }}(N)$ in Figure 1D. For a sparse distribution of vesicles, characterized by a bulk located a distance $H=100$ $\mathrm{nm}$ from the membrane, a vesicle is activated with a probability 
$p_{a c t}=0.8$ (resp. pact $=0.2$ ), when 1,200 ions are entering at a distance $100 \mathrm{~nm}$ (resp. 450 ions), and 340 ions at a distance $50 \mathrm{~nm}$ (resp. 125 ions). This result has to be compared to the 200-500 nm diameter of the AZ (Südhof, 2012). Consequently, a sparse distribution of vesicles at the $A Z$ requires a high number of entering calcium ions in order to trigger fusion, which can be achieved when channels are clustered. However, when channels are co-localized with vesicles, the activation probability $p_{a c t}$ is significantly increased: indeed 450 ions are necessary for activation for pact $=0.2$ at a distance $100 \mathrm{~nm}$. When the probability increases to 0.8 , the distance reduces to $58 \mathrm{~nm}$.

To conclude, a synapse with high release probability requires a nanometer precision of the channel location. However, this high requirement can be compensated by increasing the number of initial ions entering through VGCC clustering: with 2,000 ions, the maximum distance is relaxed to $140 \mathrm{~nm}$. On the contrary, in a pre-synaptic terminal crowded at its surface with vesicles (characterized by $H=35 \mathrm{~nm}$ ), very few initial ions are needed for an efficient release. Indeed, 50 ions are enough to activate a vesicle with probability 0.8 , wherever the channels are located in AZ (Figure 1D).

\section{COMPUTING THE DISTRIBUTION OF RELEASE PROBABILITY}

To compute the time distribution of the release probability and to account for the calcium ions at the AZ and in the bulk, a full model of the pre-synaptic terminal is needed. The main challenge for such derivation is to account both for the stochastic regime governed by rare events of individual calcium ions arriving to a sensor binding site, and the continuous description of the calcium concentration in the bulk of the synapse (Guerrier and Holcman, 2017). The classical approach consists in using partial differential equations that often cannot take into account easily the specific $\mathrm{AZ}$ organization and in particular the geometry near vesicles. An important assumption of these approaches is the use of the forward rate for the calcium to sensors. This rate is often assumed and not derived (contrary to the approach described in section 3.1).

To compute the sensor activation, a different approach is to use Monte-Carlo or Brownian simulations to follow each ionic trajectory. But this approach is often computationally greedy to detect the rare events of calcium hitting a small target (Holcman and Schuss, 2015). Recently, a hybrid Markovmass action model has been developed (Guerrier and Holcman, 2016), that combines a Markov chain to represent the stochastic events occurring at the AZ, with a mass-action laws model that represents calcium dynamics in the large bulk. The Markov chain and the mass-action model are coupled by the calcium ions coming from the bulk and binding to the sensor. The arrival time of such ions is Poissonian, with the rate computed taking into account the geometry of the vesicle, as discussed in section 3.1. This model is used to compute the time distribution of vesicular release (Guerrier and Holcman, 2016) and it shows that vesicular release is triggered by the binding of calcium ions that can originate either from the bulk or from VGCC.

The distribution of release time is bimodal although it is triggered by a single fast action potential (Figure 2). This simulation is initiated by three channels and each of them let a flow of 80 ions inside the cell during a time scale that was simulated from a Hudgkin-Huxley model (Guerrier and Holcman, 2016).

An example of specific simulation is as follows: the presynaptic terminal is a bulbous head of volume $\approx 1 \mu \mathrm{m}^{3}$. At the $\mathrm{AZ}$, we positioned eight vesicles, distributed on a square lattice of surface $\approx 0.13 \mu \mathrm{m}^{2}$ (Figure 1 and Guerrier and Holcman, 2016), so that the distance between two neighboring vesicles is $130 \mathrm{~nm}$, and each vesicle has a diameter of $40 \mathrm{~nm}$. The three calcium channels are uniformly distributed over the AZ, but remained from a distance of $6-10 \mathrm{~nm}$ (we chose around $6 \mathrm{~nm}$ here) from every vesicle. This distance corresponds to the radius of the red ribbon (Figure 3A) of height $1 \mathrm{~nm}$ the calcium ions need to reach to simulate the binding to a sensor. For each simulation, the terminal undergoes three spikes at a fixed time interval $\Delta t=$ $20--150 \mathrm{~ms}$. Once a calcium ion enters the terminal, it can either reach a vesicle with probability $p_{s}$ (Equation 3 ), or enter inside the bulk with probability $1-p_{s}$ according to the scheme of Figure 3C. We already discussed in subsection 2.2 how to chose the calcium diffusion coefficient: at the $\mathrm{AZ}$ it is $200 \mu \mathrm{m}^{2} / \mathrm{s}$, and $20 \mu \mathrm{m}^{2} / \mathrm{s}$ in the bulk to account for crowding.

Inside the bulk, ions bind to buffers with a rate constant $k_{0}=5.6 s^{-1}$ and unbind with rate $k_{-1}=500 s^{-1}$. Calcium can be extruded by pumps with a rate $k_{\text {pump }}=0.88 \mathrm{~s}^{-1}$ or they can leave the terminal with rate $k_{e s}=6.1 s^{-1}$. Finally, calcium can bind to the sensors located underneath a vesicle with a rate $k_{S}=0.3 s^{-1}$. The number of buffer molecules in the bulk varies from 0 to 1,000. A calcium ion bound at the calcium sensor can unbind with a rate $k_{U}=2000 s^{-1}$ (fast unbinding) or $5 s^{-1}$ (slow unbinding). Once 5 calcium ions are bound to a calcium sensor, then the vesicle fuses with the synaptic membrane and the vesicle spot becomes free for a new vesicle coming from the recycling pool to bind with a rate $\frac{1}{\tau_{\text {Dock }}}$ (Figure $\left.3 D\right)$. An immediate refilling of vesicles at the $\mathrm{AZ}$ is obtained with a time $\tau_{\text {Dock }}=0 \mathrm{~ms}$, but other delay are possible such as $\tau_{\text {Dock }}=50 \mathrm{~ms}$, or not refilling of vesicles with $\tau_{\text {Dock }}=\infty$ as shown in Figure 4 .

In that simulation framework, the first peak in the time distribution of vesicular release follows a single stimulation and the second one in Figure 2 (that is smaller in amplitude and wider) corresponds to the random arrival, over a much longer time period, of ions located in the synaptic terminal to small binding vesicular targets. To conclude, multiscale stochastic modeling approaches allow studying cellular events based on integrating discrete molecular events over long time scales from one milliseconds to seconds.

\subsection{Asynchronous Release}

What defines the time lag between the arrival of an action potential and the first release of a vesicle? A possible mechanism is as follows: calcium ions flow inside a channel in less than $1 \mathrm{~ms}$. We saw above that calcium ions entering from VGCG located very close to a vesicle can reach key calcium sensors in less than 

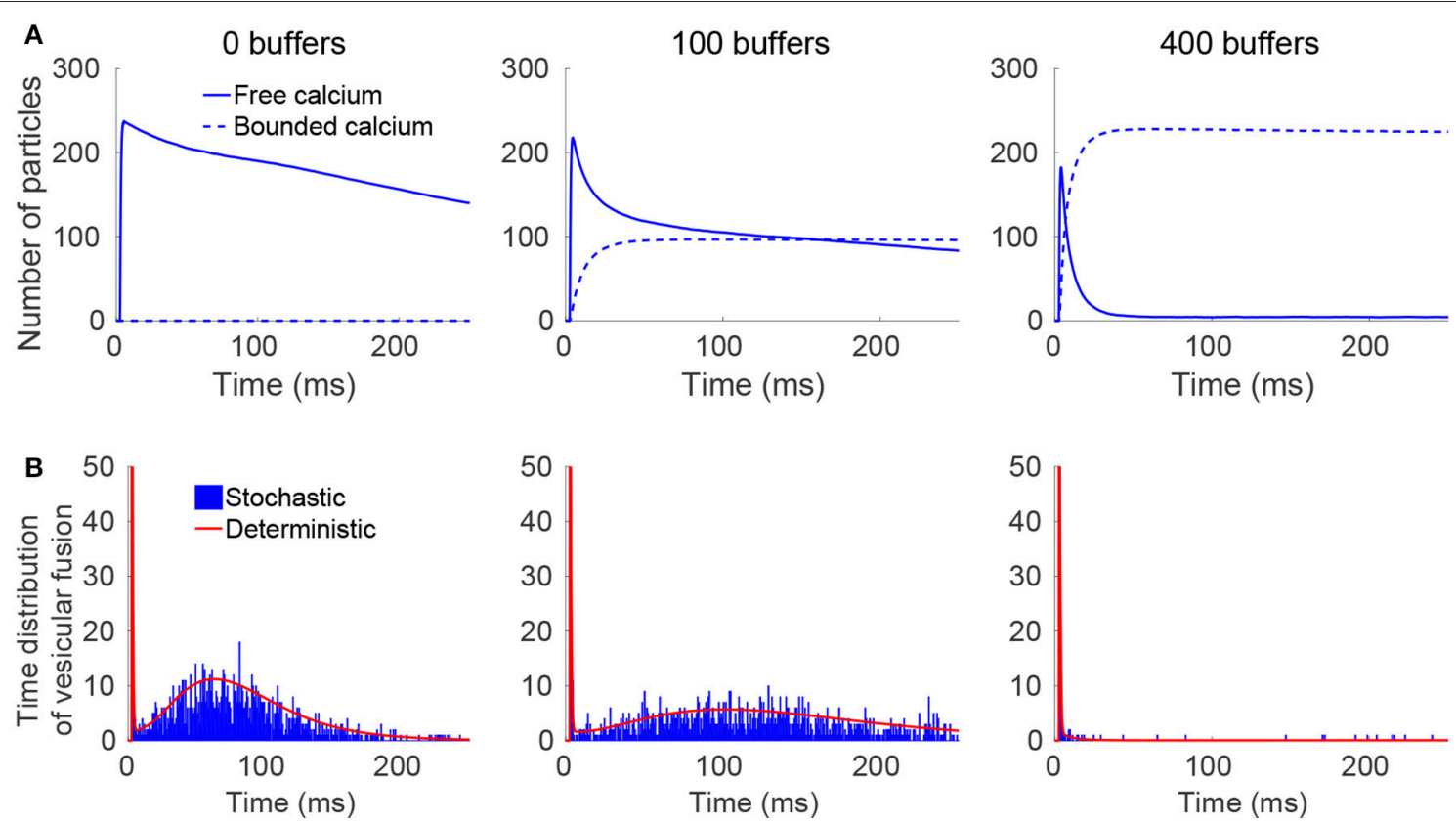

FIGURE 2 | Consequences on the release probability of calcium channel location and vesicular crowding at the AZ. Calcium time course in the pre-synaptic terminal and vesicular release activation. (A) Number of free (continuous) and buffered (dotted) ions for 0 (left), 100 (middle), and 400 (right) buffer sites. (B) Histogram of vesicular release time for the stochastic (blue), and the Markov-mass action model (red) for 0 (left), 100 (middle), and 400 (right) buffer sites.

$0.5 \mu$ s. Either all calcium binding sites necessary to trigger fusion are now activated and fusion does occur, or some sites are still empty. In this case, the release will then depend on the arrival to the ribbon underneath the vesicle of calcium ions that will have to travel from other places, such as the bulk or other VGCC located far away. This second arrival process has a rate constant of few seconds (Guerrier and Holcman, 2016). These random arrival times of calcium ions to the vesicular calcium sensors define the distribution of vesicular release that can be widely spread due to the two distributions of calcium sources (Figures 2A,B). It also reveals that the distribution contains two peaks: one generated by immediate or synchronous release, which corresponds to the case where all calcium binding sites are immediately filled (Guerrier and Holcman, 2017), and the second release, which is asynchronous over hundreds of milliseconds, the time scale of which is defined by the arrival of far away calcium ions.

In that case the cusp geometry underneath the vesicle defines the arrival rate (see above and Guerrier and Holcman, 2015). In addition, it might be possible that vesicle-tethered and cytoplasmic Syntaxin1 proteins also contribute in differentially regulating synchronous versus asynchronous release kinetics (Lee and Littleton, 2015). In that case, the asynchronous release would be determined by the vesicle-tethered mechanism and not only by calcium arrival. The two processes could also combine together.

Under the calcium hypothesis controlling asynchronous release, increasing the concentration of calcium buffers in the bulk should reduce the amount of free calcium that can travel long distance in few milliseconds. Thus, increasing calcium buffer concentration should reduce asynchronous release, as shown in numerical simulations (Figures 2A,B Guerrier and Holcman, 2016), and experimentally (Fawley et al., 2016). Indeed, this hypothesis has received more support as buffering intracellular calcium with EGTA-AM reduced asynchronous EPSC. Asynchronous or spontaneous release involve calcium coming from the bulk or VGCC located far away from the vesicles (Goswami et al., 2012; Dai et al., 2015; Stanley, 2016). The release of asynchronous vesicles was largely diminished when calcium chelation such as BAPTA (Fawley et al., 2016) was used. The authors of that study concluded that asynchronous release should rely on calcium ions involving longer trajectories compared to the ones originating from VGCC located near a vesicle. Note that the number of buffer molecules such as calmodulin in the first $100 \mathrm{~nm}$ between the vesicle and the membrane is very small (of the order of a few) and thus these molecules do not affect synchronous release, as shown also experimentally in Fawley et al. (2016). However, it remains unclear whether or not the readily releasable pool organization can influence asynchronous release.

To conclude, VGCC located underneath vesicles are not the only contributor filling the calcium binding sites, required for vesicular fusion. Actually, not all VGCC are located underneath vesicles. If one or a cluster of VGCC are not close enough or are moved away from a vesicle of a distance of 10 or $20 \mathrm{~nm}$, the probability to have the correct amount of calcium ions on the sensor binding sites can decrease significantly (Figure 1D) and thus vesicular release will have to involve calcium ions coming from far away. Modeling and experiments (Fawley et al., 2016) are now converging and suggest that this second source 


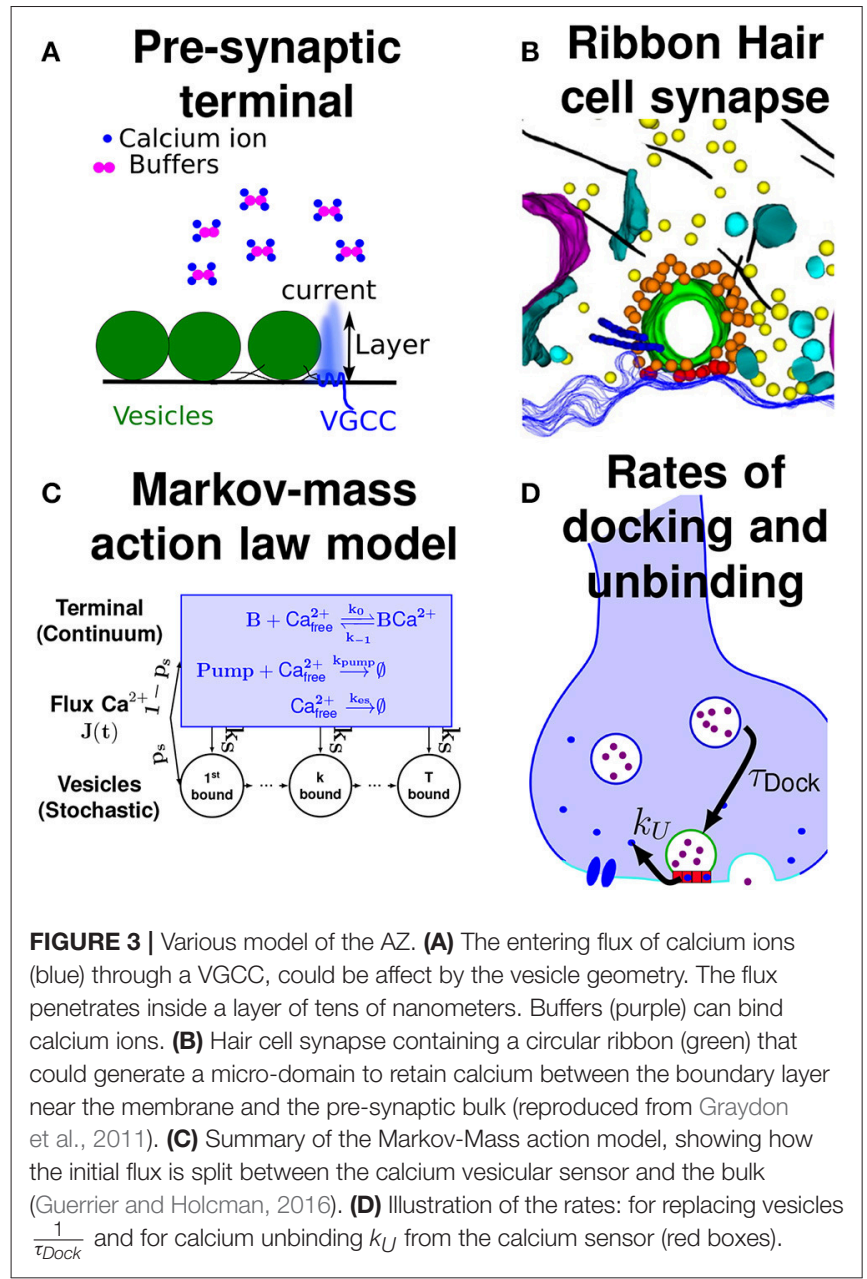

of calcium defines and regulates asynchronous release, when calcium ions generated from local VGCC is not enough. In that context, any vesicle can potentially lead to an asynchronous release as long as it does not contain enough VGCC underneath. Most likely, these vesicles are located at the periphery of the AZ, where the density of VGCC could decrease. The exact relation between VGCC distribution and vesicular organization remains unclear.

\subsection{Simulating Multiple Spikes and Paired-Pulse Ratio}

The model developed in Guerrier and Holcman (2016, 2017) can also be used to explore the short-term synaptic properties such as calcium accumulation and the distribution of time for vesicular release. First the method is consistent with any other simulation methods, second it is possible to test how the backward rate constant of the calcium ion to the sensor affects the time distribution of release, as well as the paired-pulse ratio (PPR), computed in this case as the ratio, after two consecutive spikes of the amount of fused vesicles after the second spike divided by the first one. A PPR $>1$ means that the release probability is increased which is usually interpreted as short-term synaptic facilitation, while a PPR $<1$ corresponds to a decrease, interpreted as short-term synaptic depression.

For fast calcium unbinding to the sensors, there is no accumulation of calcium in the sensor site and thus the release probability is independent of the spike train and of the buffer concentration (Figure 4A, thus PPR $=1$ ). Conversely, a slow unbinding time from the sensors is associated with a local increase in the release probability $(\mathrm{PPR}>1)$ (Figure 4B). This facilitation is due to various sources of calcium: first the ones already bound to sensors and second to calcium accumulation in the bulk following multiple spikes. This second source is diminished by increasing the amount of calcium buffers, which can lead at high buffer concentration to a decrease in the release probability PPR $<1$ (Figure 4B, bottom).

Hence, by changing the buffer concentration, a synapse can go from a facilitating state to a depressing state. To investigate the role of the readily-releasable-pool organization in the release probability, a first step is to use a vesicular replacement rate at the AZ by considering the time $\tau_{\text {Dock }}$ for a vesicle from the readily releasable pool of vesicles to replace a vesicle that has just fused: in the extreme case $\tau_{\text {Dock }}=0$, which corresponds to an immediate refilling of vesicles, and $\tau_{\text {Dock }}=\infty$ which corresponds to no refilling of vesicles: Figure 4C shows the behavior of a vesicular release with no refilling of vesicles, and a slow unbinding rate. Simulations show an increase in release probability after the second spikes, due to the calcium accumulation at the binding sites. After the third spike, the release probability is decreased, due to the lack of vesicles docked at the AZ, which would be interpreted as short-term depression. To conclude, a low unbinding rate is responsible for calcium accumulation in the sensor binding site that increases the release probability and defines short-term facilitation.

\section{CONCLUSION AND PERSPECTIVE}

The lesson from modeling and numerical simulations of diffusion in the first hundred nanometers between docked vesicles and the plasma membrane is that this boundary layer is crucial for computing the vesicular release probability due to the critical position of vesicular sensors. This space is difficult to access experimentally and its role has been underestimated in shortterm plasticity. However, experimental approaches using calcium chelator such as BAPTA or EGTA confirm the role of calcium ions traveling from far away compared to the ones entering directly through VGCC located underneath a vesicle to trigger release.

Another key feature relevant for short-term plasticity is the structural organization and the spatial correlation between the distribution of VGCCs and vesicles. Do vesicles contain the same amount of close VGCCs? What defines the exact location of VGCC underneath vesicles? Can this number fluctuate? What happens after vesicular fusion? How are VGCC redistributed? It is possible that VGCCs are constantly moving to find local optimal sites location (Schneider et al., 2015).

Short-term facilitation is classically thought as the accumulation of calcium in the synaptic bulk (calcium hypothesis 
A Fast unbind
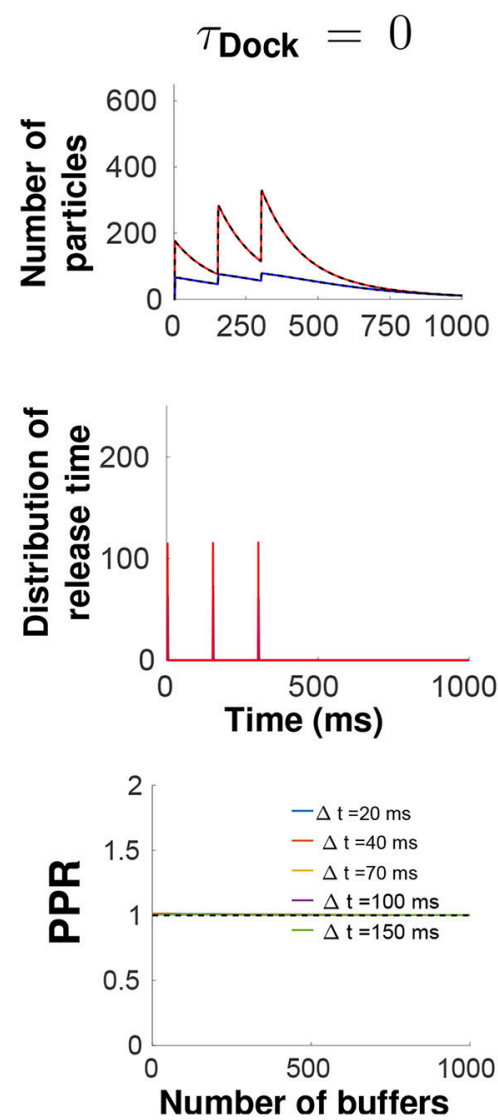

в Slow unbind

c Slow unbind
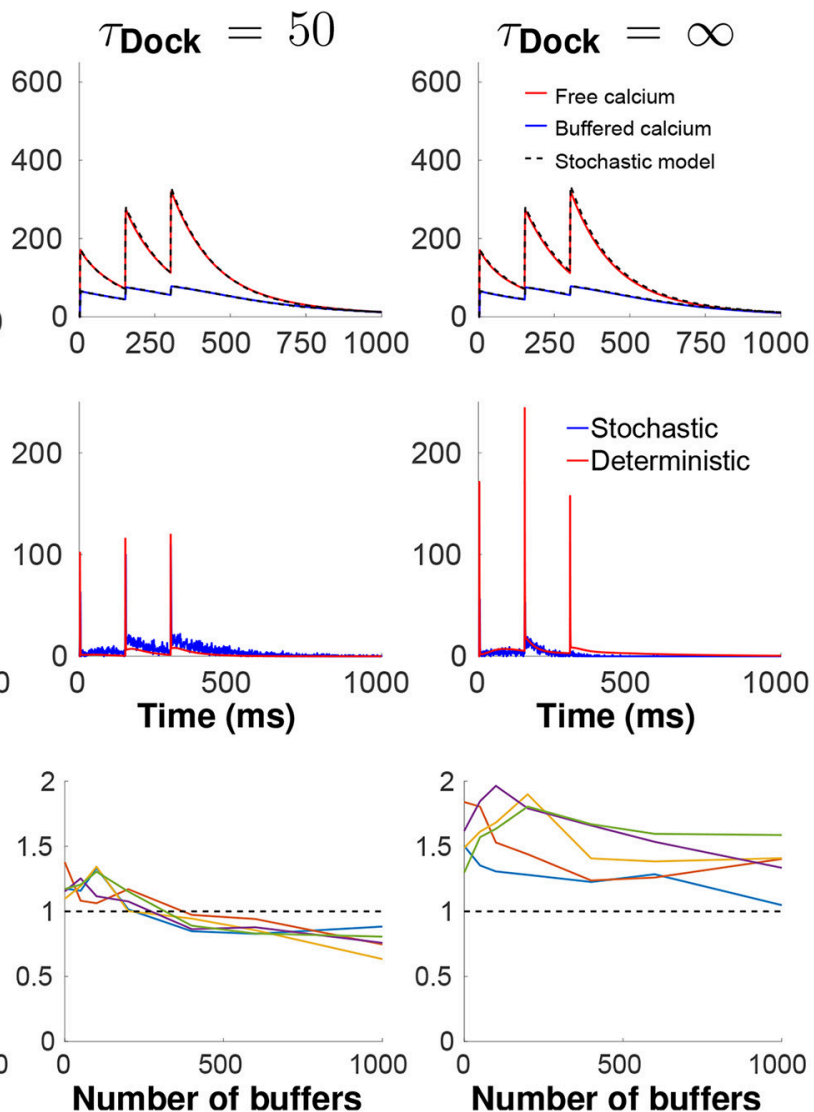

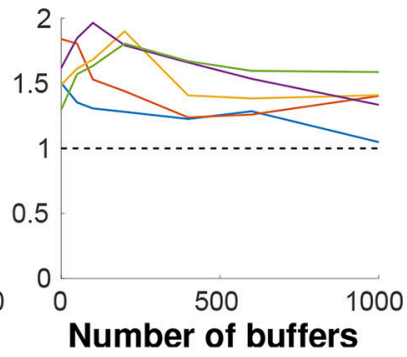

FIGURE 4 | Calcium dynamic and vesicular release in the pre-synaptic terminal following three pulses. Comparison between slow unbinding from the Snare $\left(k_{U}=\right.$ $\left.0.005 \mathrm{~ms}^{-1}\right)$ with the vesicle docking time $\tau_{D}=0 \mathrm{~ms}(\mathbf{A})$, fast unbinding $\left(k_{U}=2 \mathrm{~ms}^{-1}\right)$ with $\tau_{D}=50 \mathrm{~ms}$ (B), and fast unbinding with $\tau_{D}=\infty(\mathbf{C})$ for 100 buffer molecules and $150 \mathrm{~ms}$ between two spikes. Up: Time course of free calcium (red) and buffered calcium (blue). Middle: Probability density function of the release times. Down: Paired Pulse Ratios (PPR), computed as the ratio of the number of fused vesicles during the first and the second spike.

of Katz and Miledi, 1968), due to various possibilities such as slow and fast buffers. But computational evidences (Guerrier and Holcman, 2017) reviewed here suggest that calcium accumulation at the sensor binding sites and not in the bulk is actually the determinant effect to pre-activate vesicular release (by binding a certain fraction of the sensor sites). A similar conclusion was reached in Bornschein et al. (2013) using fluctuation analysis, calcium imaging and numerical simulation analysis indicating that the residual calcium bound to the release sensors (see also Schneggenburger and Neher, 2000), after the first AP could cause Paired Pulse Facilitation at Purkinje neuron synapses.

A byproduct of facilitation should be asynchronous release, because the random calcium accumulation at sensor increases the time window when a vesicular can be released, due to ions arriving at random time from the bulk, thus leading to a high variability in the calcium arrival and the vesicular release times. However, synaptic facilitation requires a low concentration of calcium buffer, suggesting that for facilitating synapses, calcium buffers should be maintained at a low level. In general, the relation between facilitation and asynchronous release triggered by residual calcium can be mediated by the specific molecular composition of the vesicle: indeed synaptotagmin 7 (Syt7) (Turecek and Regehr, 2018) present at cerebellar granule cell synapses onto stellate cells and Purkinje cells (mice) plays such a role: in Syt7 KO, facilitation, and asynchronous release are smaller and shorter lived than in WT, although the residual calcium was unchanged. This is in contrast with synchronous released mediated by synaptotagmin I. It would be certainly interesting to model the distribution of various synaptotagmin on vesicles to determine when a vesicle will be more likely to be released asynchronously vs synchronously. In particular, is the distribution of these two SytI and 7 different on vesicles? Do they have very different calcium buffer affinity? These properties could be explored using numerical simulations.

In terms of calcium residual regulation, a low concentration level can be achieved by preventing ER or mitochondria to come in too close proximity of the AZ. In contrast, synchronous release is associated with a high calcium buffer concentration, preventing 
calcium ions to travel from far away. More specifically, twodimensional numerical simulations (Delvendahl et al., 2015), modeling essentially the synaptic bulk, revealed that fixed endogenous buffers with low affinity, characterized by a low calcium-binding ratio, mixed with mobile buffers with high affinity, results in a fast $\mathrm{AZ}$ calcium clearance. This results in synchronous high-frequency transmission (at $200 \mathrm{~Hz}$ ). But it remains unclear how calcium fluctuations is maintained low (Modchang et al., 2010; Nadkarni et al., 2010; Dittrich et al., 2013; Weinberg, 2015) especially near the vesicular calcium sensor.

Finally, how the rate of vesicular release can vary over 6 orders of magnitude for the same synapse (Kochubey et al., 2011) also remains enigmatic. The cusp geometry and rare binding events may hold the key to the solution of this spectacular modulation of the vesicular release rate (Guerrier and Holcman, 2015).

\section{REFERENCES}

Basnayake, K., Guerrier, C., Schuss, Z., and Holcman, D. (2017). Asymptotics of extreme statistics of escape time in 1,2 and 3-dimensional diffusions. arXiv preprint arXiv:1711.01330.

Beaulieu-Laroche, L., and Harnett, M. T. (2018). Dendritic spines prevent synaptic voltage clamp. Neuron 97, 75-82.e3. doi: 10.1016/j.neuron.2017.11.016

Biederer, T., Kaeser, P. S., and Blanpied, T. A. (2017). Transcellular nanoalignment of synaptic function. Neuron 96, 680-696. doi: 10.1016/j.neuron.2017.10.006

Biess, A., Korkotian, E., and Holcman, D. (2011). Barriers to diffusion in dendrites and estimation of calcium spread following synaptic inputs. PLoS Comput. Biol. 7:e1002182. doi: 10.1371/journal.pcbi.1002182

Bornschein, G., Arendt, O., Hallermann, S., Brachtendorf, S., Eilers, J., and Schmidt, H. (2013). Paired-pulse facilitation at recurrent Purkinje neuron synapses is independent of calbindin and parvalbumin during high-frequency activation. J. Physiol. 591, 3355-3370. doi: 10.1113/jphysiol.2013.254128

Cartailler, J., and Holcman, D. (2018). Electrical transient laws in neuronal microdomains based on electro-diffusion. arXiv preprint arXiv:1803.03940.

Cartailler, J., Kwon, T., Yuste, R., and Holcman, D. (2018). Deconvolution of voltage sensor time series and electro-diffusion modeling reveal the role of spine geometry in controlling synaptic strength. Neuron 97, 1126-1136.e10. doi: 10.1016/j.neuron.2018.01.034

Courtney, N. A., Briguglio, J. S., Bradberry, M. M., Greer, C., and Chapman, E. R. (2018). Excitatory and inhibitory neurons utilize different Ca2+ sensors and sources to regulate spontaneous release. Neuron 98, 977-991.e5. doi: 10.1016/j.neuron.2018.04.022

Dai, J., Chen, P., Tianm H., and Sun, J. (2015). Spontaneous vesicle release is not tightly coupled to voltage-gated calcium channel-mediated $\mathrm{Ca} 2+$ Influx and is triggered by a $\mathrm{Ca} 2+$ sensor other than synaptotagmin-2 at the juvenile mice calyx of Held synapses. J. Neurosci. 35, 9632-9637. doi: 10.1523/JNEUROSCI.0457-15.2015

Dao Duc, K., and Holcman, D. (2010). Threshold activation for stochastic chemical reactions in microdomains. Phys. Rev. E 81:041107. doi: 10.1103/PhysRevE.81.041107

Delvendahl, I., Jablonski, L., Baade, C., Matveev, V., Neher, E., and Hallermann, S. (2015). Reduced endogenous $\mathrm{Ca} 2+$ buffering speeds active zone Ca2+ signaling. Proc. Natl. Acad. Sci. U.S.A. 112, E3075-84. doi: 10.1073/pnas.15084 19112

DiGregorio, D. A., Rothman, J. S., Nielsen, T. A., and Silver, R. A. (2007). Desensitization properties of AMPA receptors at the cerebellar mossy fiber granule cell synapse. J. Neurosci. 27, 8344-8357. doi: 10.1523/JNEUROSCI.2399-07.2007

Dittrich, M., Patillo, J. M., King, J. D., Cho, S., Stiles, J. R., and Meriney, S. D. (2013). An excess-calcium-binding-site model predicts neurotransmitter release at the neuromuscular junction. Biophys. J. 104, 2751-2763. doi: 10.1016/j.bpj.2013.05.023
Modeling vesicular trafficking and recycling at various synapses including ribbon synapses (Thoreson et al., 2016) should clarify the organization of the pre-synaptic terminal (Graydon et al., 2011, 2014) and the effect of VGCC trafficking (Schneider et al., 2015).

\section{AUTHOR CONTRIBUTIONS}

All authors listed have made a substantial, direct and intellectual contribution to the work, and approved it for publication.

\section{ACKNOWLEDGMENTS}

We thank S. Hallermann for reviewing this manuscript and for pointing us his articles, that we now discuss here. CG would like to thank the Fyssen foundation for support.

Dobramysl, U., and Holcman, D. (2018). Mixed analytical-stochastic simulation method for the recovery of a Brownian gradient source from probability fluxes to small windows. J. Comput. Phys. 355, 22-36. doi: 10.1016/j.jcp.2017. 10.058

Durand, C. M., Perroy, J., Loll, F., Perrais, D., Fagni, L., Bourgeron, T., et al. (2011). SHANK3 mutations identified in autism lead to modification of dendritic spine morphology via an actin-dependent mechanism. Mol. Psychiatry 17, 71-84. doi: 10.1038/mp.2011.57

Egles, C., Claudepierre, T., Manglapus, M. K., Champliaud, M. F., Brunken, W. J., and Hunter, D. D. (2007). Laminins containing the $\beta$-2 chain modulate the precise organization of CNS synapses. Mol. Cell. Neurosci. 34, 288-298. doi: 10.1016/j.mcn.2006.11.004

Fawley, J. A., Hofmann, M. E., and Andresen, M. C. (2016). Distinct calcium sources support multiple modes of synaptic release from cranial sensory afferents. J. Neurosci. 36, 8957-8966. doi: 10.1523/JNEUROSCI.1028-16.2016

Freche, D., Pannasch, U., Rouach, N., and Holcman, D. (2011). Synapse geometry and receptor dynamics modulate synaptic strength. PLoS ONE 6:e25122. doi: 10.1371/journal.pone.0025122

Fresche, D., Lee, C. Y., Rouach, N., and Holcman, D. (2013). Synaptic transmission in neurological disorders dissected by a quantitative approach. Commun. Integr. Biol. 5, 1-5. doi: 10.4161/cib.20818

Goswami, S. P., Bucurenciu, I., and Jonas, P. (2012). Miniature IPSCs in hippocampal granule cells are triggered by voltage-gated $\mathrm{Ca} 2+$ channels via microdomain coupling. J. Neurosci. 32, 14294-14304. doi: 10.1523/JNEUROSCI.6104-11.2012

Graydon, C. W., Cho, S., Li, G.-L., Kachar, B., and von Gersdorf, H. (2011). Sharp $\mathrm{Ca}^{2+}$ nanodomains beneath the ribbon promote highly synchronous multivesicular release at hair cell synapses. J. Neurosci. 31, 16637-16650. doi: 10.1523/JNEUROSCI.1866-11.2011

Graydon, C. W., Zhang, J., Oesch, N. W., Sousa, A. A., Leapman, R. D., and Diamond, J. S. (2014). Passive diffusion as a mechanism underlying ribbon synapse vesicle release and resupply. J. Neurosci. 34, 8948-8962. doi: 10.1523/JNEUROSCI.1022-14.2014

Gruget, C., Coleman, J., Bello, O., Krishnakumar, S. S., Perez, E., Rothman, J. E., et al. (2018). Rearrangements under confinement lead to increased binding energy of Synaptotagmin-1 with anionic membranes in $\mathrm{Mg} 2+$ and Ca2. FEBS Lett. 592, 1497-1506. doi: 10.1002/1873-3468.13040

Guerrier, C., and Holcman, D. (2017). Multiscale models and stochastic simulation methods for computing rare but key binding events in cell biology. J. Comput. Phys. 340, 617-638. doi: 10.1016/j.jcp.2017.03.058

Guerrier, C., and Holcman, D. (2014). Brownian search for targets hidden in cusplike pockets: progress and applications. Eur. Phys. J. Spec. Top. 223, 3273-3285. doi: 10.1140/epjst/e2014-02332-6

Guerrier, C., and Holcman, D. (2015). Search time for a small ribbon and application to vesicular release at neuronal synapses. Multiscale Model. Simul. 13, 1173-1193. doi: 10.1137/15M1009512 
Guerrier, C., and Holcman, D. (2016). Hybrid Markov-mass action law model for cell activation by rare binding events: application to calcium induced vesicular release at neuronal synapses. Sci. Rep. 6:35506. doi: 10.1038/srep 35506

Hodgkin, A. L., and Huxley, A. F. (1952). Currents carried by sodium and potassium ions through the membrane of the giant axon of Loligo. J. Physiol. $116,449-472$.

Holcman, D., and Schuss, Z. (2015). Stochastic Narrow Escape in Molecular an Cellular Biology, Analysis and Applications. New York, NY: Springer Verlag.

Holcman, D., and Schuss, Z. (2017). 100 years after Smoluchowski: stochastic processes in cell biology. J. Phys. A Math. Theor. 50:093002. doi: 10.1088/1751-8121/50/9/093002

Holcman, D., and Yuste, R. (2015). The new nanophysiology: regulation of ionic flow in neuronal subcompartments. Nat. Rev. Neurosci. 16, 685-692. doi: $10.1038 / \mathrm{nrn} 4022$

Katz, B., and Miledi, R. (1968). The role of calcium in neuromuscular facilitation. J. Physiol. 195, 481-492.

Keller, D., Babai, N., Kochubey, O., Han, Y., Markram, H., Schürmann, F., et al. (2015). An exclusion zone for $\mathrm{Ca} 2+$ channels around docked vesicles explains release control by multiple channels at a CNS synapse. PLoS Comput. Biol. 11:e1004253. doi: 10.1371/journal.pcbi.1004253

Kochubey, O., Lou, X., and Schneggenburger, R. (2011). Regulation of transmitter release by $\mathrm{Ca}^{2+}$ and synaptotagmin: insights from a large CNS synapse. Trends Neurosci. 34, 237-246. doi: 10.1016/j.tins.2011.02.006

Kwon, S. K., Sando, R. III, Lewis, T. L., Hirabayashi, Y., Maximov, A., and Polleux, F. (2016). LKB1 regulates mitochondria-dependent presynaptic calcium clearance and neurotransmitter release properties at excitatory synapses along cortical axons. PLoS Biol. 14:e1002516. doi: 10.1371/journal.pbio.1002516

Lee, J., and Littleton, J. T. (2015). Transmembrane tethering of synaptotagmin to synaptic vesicles controls multiple modes of neurotransmitter release. Proc. Natl. Acad. Sci. U.S.A. 112, 3793-3798. doi: 10.1073/pnas.1420312112

Modchang, C., Nadkarni, S., Bartol, T., Triampo, W., Sejnowski, T., Levine, H., et al. (2010). A comparison of deterministic and stochastic simulations of neuronal vesicle release models. Phys. Biol. 7:026008. doi: 10.1088/1478-3975/7/2/026008

Nadkarni, S., Bartol, T. M., Sejnowski, T. J., and Levine, H. (2010). Modelling vesicular release at hippocampal synapses. PLoS Comput. Biol. 6:e1000983. doi: 10.1371/journal.pcbi.1000983

Nakamura, Y., Harada, H., Kamasawa, N., Matsui, K., Rothman, J. S., Shigemoto, R., et al. (2015). Nanoscale distribution of presynaptic $\mathrm{Ca}(2+)$ channels and its impact on vesicular release during development. Neuron 85, 145-158. doi: 10.1016/j.neuron.2014.11.019

Nicoll, R. A. (2017). A brief history of long-term potentiation. Neuron 93, 281-290. doi: 10.1016/j.neuron.2016.12.015

Nielsen, T. A., DiGregorio, D. A., and Silver, R. A. (2004). Modulation of glutamate mobility reveals the mechanism underlying slow-rising AMPAR EPSCs and the diffusion coefficient in the synaptic cleft. Neuron 42, 757-771. doi: 10.1016/j.neuron.2004.04.003

Popovic, M. A., Carnevale, N., Rozsa, B., Zecevic, D. (2015). Electrical behaviour of dendritic spines as revealed by voltage imaging. Nat. Commun. 6:8436. doi: $10.1038 /$ ncomms 9436

Rothman, J. E., Krishnakumar, S. S., Grushin, K., and Pincet, F. (2017). Hypothesis - buttressed rings assemble, clamp, and release SNAREpins for synaptic transmission. FEBS Lett. 591, 3459-3480. doi: 10.1002/1873-3468.12874
Südhof, T. C. (2008). Neuroligins and neurexins link synaptic function to cognitive disease. Nature 455, 903-911. doi: 10.1038/nature07456

Südhof, T. C. (2012). The presynaptic active zone. Neuron 75, 11-25. doi: 10.1016/j.neuron.2012.06.012

Sabatini, B. L., and Regehr, W. G. (1996). Timing of neurotransmission at fast synapses in the mammalian brain. Nature 384, 170-172.

Schneggenburger, R., and Neher, E. (2000). Intracellular calcium dependence of transmitter release rates at a fast central synapse. Nature 406, 889-893. doi: $10.1038 / 35022702$

Schneggenburger, R., Han, Y., and Kochubey, O. (2012). $\mathrm{Ca}(2+)$ channels and transmitter release at the active zone. Cell Calcium 52, 199-207. doi: 10.1016/j.ceca.2012.04.011

Schneider, R., Hosy, E., Kohl, J., Klueva, J., Choquet, D., Thomas, U., et al. (2015). Mobility of calcium channels in the presynaptic membrane. Neuron 86 , 672-679. doi: 10.1016/j.neuron.2015.03.050

Sheng, M., and Kim, E. (2011). The postsynaptic organization of synapses. Cold Spring Harb. Perspect. Biol. 3:a005678. doi: 10.1101/cshperspect.a0 05678

Stanley, E. F. (2016). The nanophysiology of fast transmitter release. Trends Neurosci. 39, 183-197. doi: 10.1016/j.tins.2016.01.005

Taflia, A., and Holcman, D. (2011). Estimating the synaptic current in a multiconductance AMPA receptor model. Biophys. J. 101, 781-792. doi: 10.1016/j.bpj.2011.05.032

Tang, A. H., Chen, H., Li, T. P., Metzbower, S. R., MacGillavry, H. D., and Blanpied, T. A. (2016). A trans-synaptic nanocolumn aligns neurotransmitter release to receptors. Nature 536, 210-214. doi: 10.1038/nature 19058

Thoreson, W. B., Van Hook, M. J., Parmelee, C., and Curto, C. (2016). Modeling and measurement of vesicle pools at the cone ribbon synapse: changes in release probability are solely responsible for voltage-dependent changes in release. Synapse 70, 1-14. doi: 10.1002/syn.21871.

Turecek, J., and Regehr, W. G. (2018). Synaptotagmin 7 mediates both facilitation and asynchronous release at granule cell synapses. J. Neurosci. 38, 3240-3251. doi: 10.1523/JNEUROSCI.3207-17.2018

Von Gersdorff, H., and Borst, J. G. G. (2002). Short-term plasticity at the calyx of held. Nat. Rev. Neurosci. 3, 53-64. doi: 10.1038/n rn705

Weinberg, S. H. (2015). Fluctuations in calcium concentration alter the temporal dynamics of calcium-dependent signaling cascades. Biophys. J. 108:178a. doi: 10.1016/j.bpj.2014.11.982

Xie, X., Liaw, J. S., Baudry, M., Berger, T. W. (1997). Novel expression mechanism for synaptic potentiation: alignment of presynaptic release site and postsynaptic receptor. Proc. Natl. Acad. Sci. U.S.A. 94, 6983-6988.

Conflict of Interest Statement: The authors declare that the research was conducted in the absence of any commercial or financial relationships that could be construed as a potential conflict of interest.

Copyright (c) 2018 Guerrier and Holcman. This is an open-access article distributed under the terms of the Creative Commons Attribution License (CC BY). The use, distribution or reproduction in other forums is permitted, provided the original author(s) and the copyright owner(s) are credited and that the original publication in this journal is cited, in accordance with accepted academic practice. No use, distribution or reproduction is permitted which does not comply with these terms. 